\title{
Experimental Investigation on Wire-Electro Discharge Machining of Tungsten Carbide (WC) using Response Surface Methodology (RSM)
}

\author{
V.P. Srinivasan ${ }^{1, *}$ and P.K. Palani ${ }^{2, \dagger}$ \\ ${ }^{1}$ Sri Krishna College of Engineering and Technology, Coimbatore, Tamil Nadu, India \\ ${ }^{2}$ Associate Professor, Government College of Technology, Coimbatore, Tamil Nadu, India
}

Corresponding Author Email: *vpsrinivasa@gmail.com, ${ }^{\dagger}$ pkpalaniku@gmail.com

\begin{abstract}
In this work, Wire-Electro Discharge Machining (WEDM) of Tungsten Carbide (WC) was carried out using copper wire-electrode of diameter $0.25 \mathrm{~mm}$. Diatomite powder mixed with distilled water is used as the dielectric fluid to increase the working fluid conductivity. Selection of appropriate machining parameters in WEDM is one of the most important aspects taken into consideration as these conditions to determine the important characteristics such as Material Removal Rate (MRR) and surface roughness (Ra) among others. The main machining parameters such as voltage $(V)$, pulse-on time $\left(T_{o n}\right)$ and wire tension $(W T)$ were chosen to determine listed technological characteristics. The characteristic features of the WEDM process are explored through Response Surface Methodology (RSM) based on Design of Experiments (DOE). From the results, it is evident that the pulse-on time is the most significant factor followed by the voltage and wire tension.
\end{abstract}

Keywords: WEDM, Tungsten Carbide, Material Removal Rate, surface roughness, RSM, DOE

Received: June-29-2018, Accepted: January-19-2019, https://doi.org/10.14447/jnmes.v22i3.a07

\section{INTRODUCTION}

Wire-Electro Discharge Machining is a special thermo machining process which is capable of machining parts with varying hardness and complex shapes very accurately, also parts with sharp edges that are very difficult to machine by the main stream machining processes. WEDM is a widely accepted unconventional material removal process used to manufacture components which have intricate shapes and profiles. The conventional EDM which uses an electrode to initialize the sparking process is adapted in WEDM process. To achieve very small corner radii, a continuously travelling wire-electrode made of thin copper, brass or tungsten of diameter 0.05 to $0.3 \mathrm{~mm}$ is utilized in the WEDM process. The wire used in WEDM is kept in tension using a mechanical device to produce accurate parts. Also, WEDM does not make direct contact between the wire-electrode and the workpiece eliminating mechanical stresses and chatter problems during machining. The material removal mechanisms of EDM and WEDM are identical whereas, the functional characteristics are different. EDM uses toolelectrode to machine the workpiece and WEDM uses thin wire (wireelectrode) which is continuously feed through the workpiece to the machine with high accuracy. The microprocessor constantly maintains 0.025 to $0.05 \mathrm{~mm}$ gap between the wire-electrode and the workpiece [1-3]. Micro-WEDM has become prominent as the popular micromachining processes for fabrication of micro-parts. The predominant problems faced in this processes are the poor surface finish and the low machining rate. To improve the performance of the micro-WEDM, the low-frequency workpiece vibration assistance can be provided to enhance the flushing conditions and to reduce the adhesion of the wireelectrode and the workpiece [4]. To produce micro-parts with WEDM, ultra thin wire-electrodes of diameters $20,25,30$ and $50 \mu \mathrm{m}$ can be used [5]. The residual stress generated on the workpiece during the WEDM process should be low as possible to achieve good surface integrity and longer service life. However, the residual stress formation depends on setting machining parameters and the material to be machined [6]. The WEDM cut surface of the workpiece have poor surface integrity and decreased fatigue life as those compared with the polished surface [7]. At the idle voltage, pulse-on time, and the discharge current the crater dimensions are not influenced. Crater developed during machining does not have uniform shape with same machining parameters [5]. Deep craters are formed on the machined surface due to more frequent melting explosion caused by high discharge energy [8]. The surface roughness increases with increase in pulse-on time and pulse-peak current while machining hot-pressed boron carbide and newly developed DC53 die steel in WEDM [8-9]. Also, surface roughness has different variations while machining ceramic particulate reinforced Al matrix composites in WEDM. The surface roughness decreases in $\mathrm{Al} / \mathrm{SiC}_{\mathrm{p}}$ composites as the volume fraction of $\mathrm{SiC}$ is increased and in $\mathrm{Al} / \mathrm{Al}_{2} \mathrm{O}_{3 \mathrm{p}}$ the surface roughness increases as the volume fraction of alumina increased. Volume fractions, size of ceramic reinforcements, coefficient of thermal expansion, heat fusion, thermal diffusivity and melting temperature are significant factors on surface finish of particulate reinforced $\mathrm{Al}$ matrix composites [10]. The workpiece material with a low melting temperature and specific heat exhibits high MRR in WEDM [11]. In $\mu$-WEDM of gold-coated Si wafer, the MRR can be enhanced from $1 \%$ to $33 \%$ by nanopowder mixed dielectric fluid medium [12]. To facilitate effective machining of Tungsten Carbide, various conductive powder particles like graphite and diatomite are mixed separately with the dielectric fluid medium to increase the micro-hardness $(\mu-\mathrm{H})$, MRR and to reduce the surface roughness [13-14]. Tungsten Carbide is categorized under extremely hard and difficult-to-cut material used widely in manufacturing because of its high wear, abrasion and corrosion resistance. Tungsten Carbide has extreme applications in manufacturing carbide dies, cutting tools and forestry tools [15-16]. About $50 \%$ of all the carbide production is utilized for the machining applications and the cemented carbides are being increasingly used for 


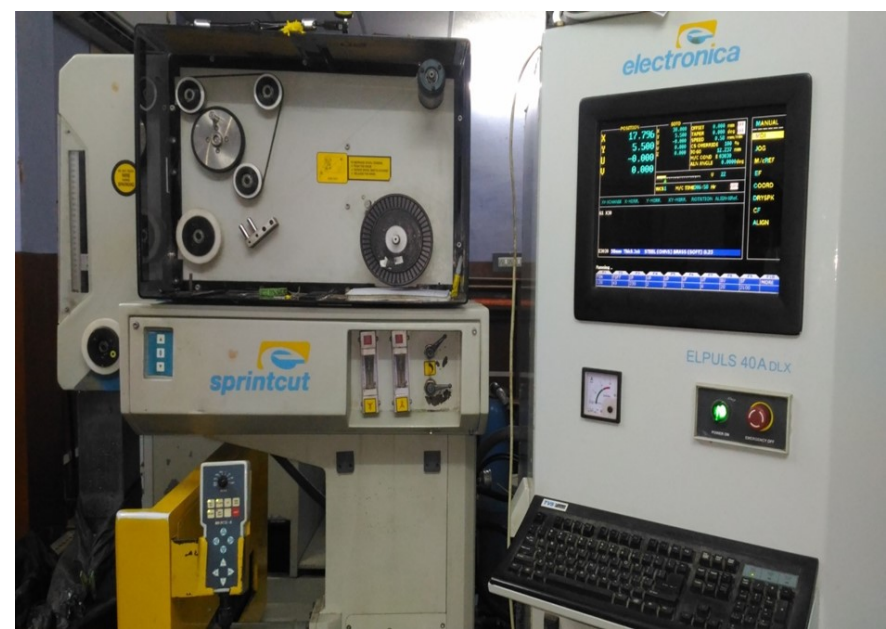

Fig. 1. ELECTRONICA SPRINTCUT ELPLUS 40A DLX WEDM machine

the non-machining applications such as mining, oil and gas drilling, metal forming and forestry tools [17]. Even though Tungsten Carbide has extreme wear and thermal properties, at relatively moderate temperature they are susceptible to oxidation [18] and by the diffusion impregnation with silicon, this material can be rendered oxidation resistant [19]. The RSM is the collection of mathematical and statistical procedures for empirical modeling approach to examine the relationship between various process parameters and their responses with the various desired criteria and identifying the significance of these processes on the coupled responses [20]. The primary influencing factors like pulse peak current, pulse duration, pulse-off period, wire feed, wire tension and flushing pressure have effect on the productivity and on the surface quality of machined components. Optimal machining conditions can be obtained for maximization of both the Material Removal Rate and the surface finish by developing models using the non-linear regression method [21]. By increasing the average gap voltage, the cutting speed will decrease and the kerf width will increase on the other hand by increasing the pulse-on time, both the cutting speed and kerf width will increase [22]. By employing Grey relational analysis, the response characteristics such as Material Removal Rate, surface roughness, and gap current can be improved with 6\% error [23]. Among other parameters, the pulse-on time has an imperative effect on Material Removal Rate and surface roughness [24]. From the literature survey, it is evident that in the WEDM process the pulse-on time is significant parameter among others. Also no researchers have attempted to machine the Tungsten Carbide with copper wire-electrode of diameter $0.25 \mathrm{~mm}$ using diatomite power mixed dielectric fluid medium in WEDM and hence an attempt is made to find the increased MRR and decreased Ra.

\section{MATERIALS AND METHODS}

\subsection{Workpiece, wire-electrode and dielectric fluid}

The workpiece material used in this work was Tungsten Carbide of cylindrical shape with the dimensions $23 \mathrm{~mm}$ diameter and $87 \mathrm{~mm}$ length. The properties of Tungsten Carbide material is elucidated in Table 1. The wire-electrode used in this work was copper wire of diameter $0.25 \mathrm{~mm}$ and the dielectric fluid used in this work was diatomite powder-mixed deionised water. The diatomite powder is used because it is gently abrasive and insoluble in water and also the cost of diatomite powder is lower to that of other metal powders. The diatomite powder-mixed dielectric fluid produces increased MRR [25].

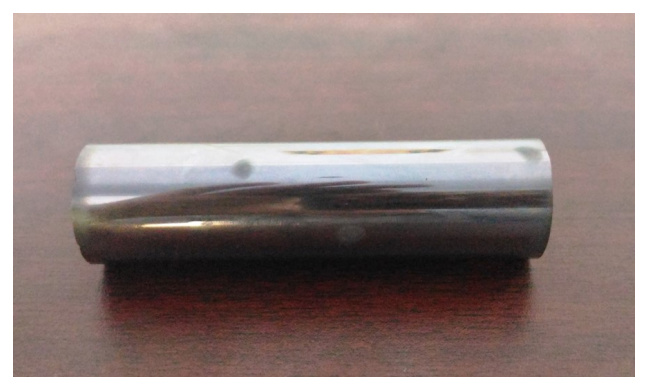

Fig. 2. Tungsten Carbide workpiece before machining

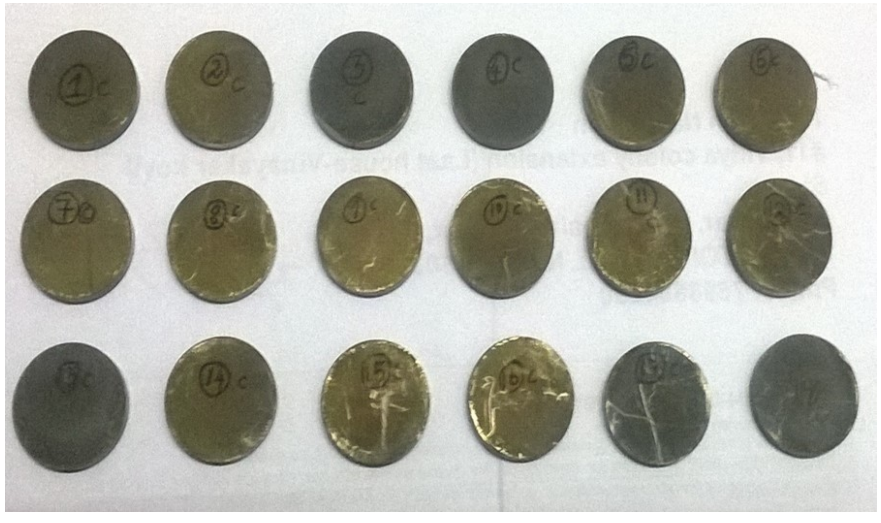

Fig. 3. Tungsten Carbide workpiece after machining

\subsection{Experimental procedure}

In this work, ELECTRONICA SPRINTCUT ELPLUS 40A DLX series machine was used to carry out experiments as shown in Fig. 1. The following steps are carried out to machine the workpiece.

- The workpiece is set in the vice, using dial gauge its straightness is checked and the co-ordinates of the machine are set to zero.

- The combinations of input parameters are feed into the machine as per the DOE.

- The high pressure flushing setup is then switched ON.

- After machining, the machine automatically alarms indicating completion.

- Then the MRR is calculated by using the formulae.

- This procedure is repeated for remaining trials.

The Tungsten Carbide workpiece before machining and after machining are shown in Fig. 2 and Fig. 3 respectively.

\subsection{DOE}

The DOE is a systematic approach to determine the relationship between factors affecting the process and the response of that process. It enables to obtain useful information about the process by conducting a very minimal number of experiments [26]. As per the DOE, the experi-

Table 1. Properties of Tungsten Carbide

\begin{tabular}{cc}
\hline Essential Properties & Description \\
\hline Chemical Formula & $\mathrm{WC}$ \\
Density & $15.80 \mathrm{~g} / \mathrm{cm}^{3}$ \\
Melting Point & $2870^{\circ} \mathrm{C}$ \\
Boiling Point & $6000^{\circ} \mathrm{C}$ \\
Electrical Resistivity & $2 \times 10^{7}(\Omega \mathrm{m})$ \\
Young's Modulus (E) & $550 \mathrm{Gpa}$ \\
\hline
\end{tabular}


ments were conducted with three factors varied at three levels as shown in Table 2. The table design consisting of 18 experiments based on Box-Behnken design method was generated using DESIGN EXPERT 7.0.0 statistical software. The experimental results for Material Removal Rate and surface roughness are shown in Table 3.

\section{RESULTS AND DISCUSSION}

Experiments were conducted to optimize the WEDM parameters for Tungsten Carbide workpiece. In the present work, three major factors like voltage, pulse-on time and wire tension were considered as critical parameters and varied at three levels.

From the Table 4, it is found that when the pulse-on time and voltage are maximum i.e., $130 \mu$ s and $230 \mathrm{~V}$ respectively and minimum wire tension of $2 \mathrm{gms}$, the MMR was found to be $4.99408 \mathrm{~mm}^{3} / \mathrm{min}$ and it is

Table 2. Experimental factors and their levels for WEDM of Tungsten Carbide

\begin{tabular}{ccccc}
\hline Factors & $\begin{array}{c}\text { Pulse-on time } \\
\left(\mathrm{T}_{\text {on }}\right)\end{array}$ & $\begin{array}{c}\text { Voltage } \\
(\mathrm{V})\end{array}$ & $\begin{array}{c}\text { Wire Tension } \\
(\mathrm{WT})\end{array}$ \\
\hline \multicolumn{2}{c}{ Units } & $\mu \mathrm{\mu sec}$ & volts & gms \\
\hline \multicolumn{2}{c}{ Designation } & $\mathrm{A}$ & $\mathrm{B}$ & $\mathrm{C}$ \\
\hline \multirow{2}{*}{ Level } & 1 & 110 & 170 & 2 \\
& 2 & 120 & 200 & 6 \\
& 3 & 130 & 230 & 10 \\
\hline
\end{tabular}

Table 3. Experimental results for MRR and Ra of Tungsten Carbide

\begin{tabular}{|c|c|c|c|c|c|}
\hline \multirow{2}{*}{ Run } & \multicolumn{3}{|c|}{ Factors } & \multirow{2}{*}{$\begin{array}{c}\text { MRR } \\
\left(\mathrm{m}^{3} / \mathrm{min}\right)\end{array}$} & \multirow{2}{*}{$\begin{array}{c}\text { Surface Rough- } \\
\text { ness } \\
\text { (mm) }\end{array}$} \\
\hline & A & B & $\mathrm{C}$ & & \\
\hline 1 & 120 & 230 & 10 & 3.7096 & 1.2 \\
\hline 2 & 120 & 200 & 6 & 4.3725 & 1.32 \\
\hline 3 & 110 & 200 & 2 & 2.2097 & 1.01 \\
\hline 4 & 110 & 200 & 10 & 2.663 & 1.1 \\
\hline 5 & 120 & 170 & 2 & 2.9674 & 1.21 \\
\hline 6 & 120 & 170 & 10 & 3.462 & 1.39 \\
\hline 7 & 130 & 200 & 10 & 4.924 & 1.45 \\
\hline 8 & 130 & 200 & 2 & 4.5156 & 1.28 \\
\hline 9 & 120 & 200 & 6 & 3.462 & 1.29 \\
\hline 10 & 120 & 200 & 6 & 4.77 & 1.47 \\
\hline 11 & 130 & 230 & 6 & 4.924 & 1.49 \\
\hline 12 & 120 & 230 & 2 & 3.8466 & 1.2 \\
\hline 13 & 110 & 230 & 6 & 2.5965 & 1.1 \\
\hline 14 & 120 & 200 & 6 & 4.1544 & 1.41 \\
\hline 15 & 120 & 200 & 6 & 4.1544 & 1.42 \\
\hline 16 & 130 & 170 & 6 & 3.1472 & 1.009 \\
\hline 17 & 110 & 170 & 6 & 2.0772 & 0.8 \\
\hline 18 & 110 & 170 & 6 & 1.888 & 0.7 \\
\hline
\end{tabular}

Table 4. Response optimization for MRR and Ra of Tungsten Carbide

\begin{tabular}{|c|c|c|c|c|c|c|}
\hline S.No & $\begin{array}{l}\text { Pulse-on time }\left(\mathrm{T}_{\text {on }}\right) \text { in } \\
\mu \mathrm{sec}\end{array}$ & Voltage (V) in volts & $\begin{array}{l}\text { Wire Tension (WT) in } \\
\text { gms }\end{array}$ & $\begin{array}{l}\text { Material Removal Rate } \\
\text { (MRR) in } \mathrm{m}^{3} / \mathrm{min}\end{array}$ & $\begin{array}{l}\text { Surface Rough- } \\
\text { ness }(\mathrm{Ra}) \text { in } \mathrm{mm}\end{array}$ & Desirability \\
\hline 1 & 130 & 230 & 2 & 4.9940 & 1.0290 & 0.8978 \\
\hline 2 & 130 & 229.61 & 2 & 4.9971 & 1.0306 & 0.8958 \\
\hline 3 & 129.88 & 230 & 2 & 4.9884 & 1.0356 & 0.8956 \\
\hline 4 & 130 & 230 & 2.04 & 4.9929 & 1.0384 & 0.8946 \\
\hline 5 & 129.54 & 230 & 2 & 4.9716 & 1.0459 & 0.8901 \\
\hline 6 & 130 & 230 & 2.13 & 4.9901 & 1.0420 & 0.8874 \\
\hline 7 & 130 & 230 & 2.18 & 4.9888 & 1.0411 & 0.8839 \\
\hline 8 & 128.81 & 229.93 & 2 & 4.9308 & 1.0428 & 0.8780 \\
\hline 9 & 130 & 230 & 2.46 & 4.9806 & 1.0551 & 0.8612 \\
\hline 10 & 130 & 230 & 2.59 & 4.9771 & 1.0582 & 0.8506 \\
\hline 11 & 127.18 & 230 & 2 & 4.8118 & 1.0593 & 0.8486 \\
\hline 12 & 130 & 215.48 & 2 & 4.9342 & 1.0617 & 0.8335 \\
\hline 13 & 130 & 212.22 & 2.36 & 4.8708 & 1.1286 & 0.7936 \\
\hline 14 & 123.09 & 230 & 2 & 4.3599 & 1.0947 & 0.7785 \\
\hline 15 & 130 & 229.94 & 3.44 & 4.9572 & 1.0816 & 0.7776 \\
\hline 16 & 130 & 199.68 & 2 & 4.4752 & 1.011 & 0.7627 \\
\hline 17 & 121.9 & 230 & 2 & 4.1873 & 1.0816 & 0.7583 \\
\hline 18 & 122.19 & 199.95 & 2 & 4.2839 & 1.1274 & 0.6457 \\
\hline
\end{tabular}

the most significant value as compared to other values. Fig. 4 indicates the impact on MRR by other parameters. It is observed that when the pulse-on time increases gradually, MRR also increases gradually.

From the Table 4, it is found that when the pulse-on time and voltage are maximum i.e., $130 \mu$ s and $230 \mathrm{~V}$ respectively and minimum wire tension of $2 \mathrm{gms}$, the surface roughness was found to be $1.0290 \mathrm{~mm}$ and it is the most significant value as compared to other values. Fig. 5 indicates the impact on surface roughness by other parameters. It is observed that when the pulse-on time increases, the surface roughness also increases at starting stage but, to a certain extent it decreases.

\section{CONCLUSIONS}

The parameters affecting the Wire-Electro Discharge Machining of Tungsten Carbide were studied. The optimum parameter combinations for maximizing the Material Removal Rate and minimizing the surface roughness were identified. The parameters like voltage, pulse-on time and wire tension have the significant effect on the MRR and Ra. It is identified that among all parameters, the pulse-on time is the most significant parameter.

In this study, the maximum MRR is obtained for pulse-on time - 130 $\mu \mathrm{s}$, voltage $-230 \mathrm{~V}$ and wire tension $-6 \mathrm{gms}$ and the minimum the surface roughness is obtained for pulse-on time - $110 \mu$ s, voltage - 170 $\mathrm{V}$ and wire tension -6 gms.

\section{REFERENCES}

[1] K.H. Ho, S.T. Newman, S. Rahimifard, and R.D. Allen, International Journal of Machine Tools and Manufacture, 44, 1247 (2004).

[2] E.C. Jameson, Society of Manufacturing Engineers, Dearbern, Michigan, 16 (2001).

[3] G.F. Benedict, "Electrical discharge machining (EDM)", NonTraditional Manufacturing Processes, Marcel Dekker, Inc, New York \& Basel, 231(1987).

[4] Deepak Rajendra Unune, and Harlal Singh Mali, Engineering Science and Technology, 20, 222 (2017).

[5] F. Klocke, D. Lung, D. Thomaidis, and G. Antonoglou, Journal of Materials Processing Technology, 149, 579 (2004).

[6] Pujari Srinivasa Rao, Koona Ramji, and Beela Satyanarayana, Alexandria Engineering Journal, 55, 1077 (2016).

[7] Zhe Chen, Johan Moverare, Ru Lin Peng, and Sten Johansson, Procedia CIRP, 45, 307 (2016).

[8] Ravindranadh Bobbili, V. Madhu, and A.K. Gogia, Defence Technology, 11, 344 (2015). 
[9] K. Kanlayasiri, and S. Boonmung, Journal of Materials Processing Technology, 187-188, 26 (2007).

[10]Nilesh G. Patil, and P.K. Brahmankar, Procedia CIRP, 42, 280 (2016).

[11]Ravindranadh Bobbili, V. Madhu, and A.K. Gogia, Engineering Science and Technology, 18, 664 (2015).

[12]S. Jarin, T. Saleh, M. Rana, A.G.A. Muthalif, and M.Y. Ali, Procedia Engineering, 184, 171 (2017).

[13]Jagdeep Singh, and Rajiv Kumar Sharma, Perspectives in Science, 8, 455 (2016).

[14]V.P. Srinivasan, and P.K. Palani, Journal of Applied Sciences Research 11(22), 24 (2015).

[15]M.P. Jahan, Y.S. Wong, and M. Rahman, Journal of Materials Processing Technology, 209, 1706 (2009).

[16]M.P. Jahan, M. Rahman, and Y.S. Wong, International Journal of Machine Tools and Manufacture, 51, 837 (2011).

[17]I. Puertas, C.J. Luis, and L. Álvarez, Journal of Materials Processing Technology, 153-154, 1026 (2004).

[18]Samuel A. Humphry-Baker, Ke Penga, and William E. Lee, International Journal of Refractory Metals and Hard Materials, 66, 135 (2017).

[19]B. Casas, X. Ramis, M. Anglada, J.M. Salla, and L. Llanes, International Journal of Refractory Metals and Hard Materials, 19, 303 (2001).

[20]R.H. Myers and D.H. Montgomery, "Response Surface Methodology", John Wiley \& Sons, USA., (1995).

[21]Kapil Kumar, and Sanjay Agarwal, International Journal of Advanced Manufacturing Technology, 62, 617 (2012).

[22]Probir Saha, Debashis Tarafdar, Surjya K. Pal, Partha Saha, Ashok K. Srivastava, and Karabi Das, Applied Soft Computing, 13, 2065 (2013).

[23]Ravindranadh Bobbili, V. Madhu, and A.K. Gogia, Engineering Science and Technology, an International Journal, 18, 720 (2015).

[24]Somvir Singh Nain, Dixit Garg, and Sanjeev Kumar, Engineering Science and Technology, 20, 247 (2017).

[25]J.M. Muniu, B.W. Ikua, D.M. Nyaanga, and S.N. Gicharu, Proceedings of the 2012 Mechanical Engineering Conference on Sustainable Research and Innovation, 4, 280 (2012).

D.C. Montgomery, "Design and analysis of experiments", Wiley (New York), ISBN: 0-471-15746-5, (1997). 